\title{
Ultrasonic Flaw Detection and Imaging through Reverberant Layers via Subspace Analysis and Projection
}

\author{
Ramazan Demirli, ${ }^{1}$ Moeness G. Amin, ${ }^{1}$ Xizhong Shen, ${ }^{2}$ and Yimin D. Zhang1 \\ ${ }^{1}$ Center for Advanced Communication, Villanova University, Villanova, PA 19085, USA \\ ${ }^{2}$ School of Electrical and Electronic Engineering, Shanghai Institute of Technology, \\ Shanghai 201418, China
}

Correspondence should be addressed to Ramazan Demirli, ramazan.demirli@villanova.edu

Received 12 March 2012; Revised 30 May 2012; Accepted 31 May 2012

Academic Editor: Erdal Oruklu

Copyright (c) 2012 Ramazan Demirli et al. This is an open access article distributed under the Creative Commons Attribution License, which permits unrestricted use, distribution, and reproduction in any medium, provided the original work is properly cited.

\begin{abstract}
Ultrasonic flaw detection and imaging through reverberant layers are challenging problems owing to the layer-induced reverberations and front surface reflections. These undesired signals present a strong clutter and mask the flaw echoes. In this paper, a subspace-based approach is developed for removing, or significantly reducing, the unwanted reverberations, enabling proper flaw detection and imaging. The technique utilizes a set of independent clutter-only reference measurements of the material through the layer. If these measurements are not available, array measurements of the material with flaws are used instead. The clutter, due to its high strength relative to the flaw reflections, forms a subspace spanned by the eigenvectors corresponding to the dominant eigenvalues of the data covariance matrix. The clutter subspace is estimated and removed using orthogonal subspace projection. The clutter usually occupies multidimension subspace that is dependent on the level of coupling, material inhomogeneity, surface roughness, and the sampling rate of the measurements. When the clutter-only reference is not available, information theoretic techniques are used to estimate the dimension of the clutter subspace so that clutter signals are sufficiently suppressed without distorting the flaw signals. The effectiveness of the proposed approach is demonstrated using simulations and real measurement results.
\end{abstract}

\section{Introduction}

Ultrasonic detection and imaging of flaws through a layer or screen are challenging problems encountered in ultrasound nondestructive evaluation (NDE). In particular, industrial materials are often manufactured in the forms of multiple layers, which present strong reflections at layer interfaces when exposed to ultrasound testing. These reflections usually repeat themselves in the course of an ultrasonic measurement, giving rise to strong and repeating reverberation patterns [1]. In medical ultrasound, direct access to the tissue of interest is not always possible, and hence ultrasonic measurements are often performed through another tissue or anatomic structure [2]. For example, in ultrasound imaging of brain for abnormalities or tumors, measurements are performed through the skull, which presents strong reverberation signal.
Reverberation signals induced by the top layer (i.e., imaging screen) often mask the target echoes and make the detection and localization of material flaws or tissue abnormalities extremely difficult, if not impossible. Therefore, such reverberation signals (which are also referred to hereafter as reverberation clutter, or simply clutter) must be suppressed or sufficiently mitigated in order to reveal the target echoes. The majority of the existing approaches dealing with reverberation are based on the ideal acoustic wave propagation model in the layered media $[1,3-6]$. For example, Saniie and Nagle have developed analytical models of reverberation patterns measured from multilayered media [1]. These models are used for the classification of echoes associated with each layer. The predictive deconvolution technique [7], commonly used in reverberation suppression in seismic explorations, has been applied to ultrasound reverberation suppression $[3,4]$. This method also assumes, 
although implicitly, an ideal propagation model by relying on the repeatability of reverberating patterns. The methods presented in $[5,6]$ deal with the identification of reverberation echoes in multilayered media based on time-of-flight analysis of all possible echoes and their power spectrum comparison. In addition to the ideal propagation models mentioned above, these techniques assume nonoverlapping echo patterns amenable to time-of-flight analysis, which requires the thickness of each layer to be relatively large compared to the echo wavelength. Further, the existing approaches often deal with ultrasonic measurements in the far field of the transducer and, as such, use immersion techniques. These techniques are not practical for field testing scenarios in ultrasonic NDE where only contact measurements can be performed in the near field of the transducer. Making contact measurements through the layer is further complicated by the coupling issues and strong irregular echoes from the layer front surface. The reverberation patterns in these cases cannot be simply predicted and removed from the measurements.

Among different possible approaches to considerably attenuate clutter, direct subtraction of background response signal, measured in empty reference scenes, from the response signal with targets (flaws) is commonly used in radar signal processing [8]. Direct application of this method to practical ultrasonic reverberation suppression, however, proves inefficient, due to the significant variations of ultrasound measurements due to the coupling, material inhomogeneity, and surface roughness.

In this paper, we propose an alternative approach based on reverberation subspace learning and projection. This approach has been recently used in through-the-wall radar imaging to remove the wall clutter and enhance the visibility of indoor targets $[9,10]$ and also in ground penetrating radar for landmine detection $[8,11]$. In the proposed approach, the clutter is removed by projecting the received signal onto a subspace that is orthogonal to the bases of possible clutter responses. To construct a comprehensive clutter subspace for efficient clutter removal, we consider two cases of reverberation subspace learning that are of interest to ultrasound NDE, namely, (i) reference-based subspace learning (offline) utilizing a set of flaw-free reverberation measurements and (ii) reference-free subspace learning directly from the online array data. The latter is of particular importance in NDE array imaging when access to a healthy replica is not available [12]. In the first case, we make use of a few independent reverberation measurements from selected locations using a healthy replica of the test material. Then, we construct a clutter subspace utilizing a shift-based perturbation model to account for variations due to surface contacts, material inhomogeneity, and surface roughness. In the second case, we use a physical or synthetic aperture array and form the clutter subspace by exploiting the spatial coherency of the reverberation signals and incoherency of the flaw echoes in the array data. In both cases, the clutter often occupies multiple dimensions of the subspace, dependent on the level of coupling, material inhomogeneity, surface roughness, and the sampling rate of the measurements. Therefore, the dimension of the clutter subspace must be properly estimated before the orthogonal projection is applied. Underestimation of the clutter subspace may result in insufficient clutter removal. On the other hand, particularly in the second case where the flaw signals are present in the clutter subspace construction, overestimation of the clutter subspace will result in flaw signal removal. To avoid this problem, information theoretic techniques are used to estimate the dimension of the clutter subspace so that clutter signals are sufficiently suppressed without distorting the flaw signals.

The effectiveness of the proposed method is examined and demonstrated using both simulations and real experiment data. The results clearly show that strong clutter can be significantly suppressed using the proposed technique based on subspace learning and orthogonal subspace projection. For comparison, we also apply the background subtraction and predictive deconvolution techniques, which show inferior performance to the proposed technique in the presence of irregular variations.

The remainder of the paper is organized as follows. Section 2 presents a clutter subspace construction and projection technique utilizing flaw-free reference measurements of reverberation. Section 3 presents a reference-free clutter subspace construction and projection technique based on transducer array data. Section 4 presents the simulation and experimental results of the clutter removal techniques in both scenarios.

\section{Clutter Removal Using Reference Reverberation Measurements}

2.1. The Proposed Technique. Consider a flaw detection and imaging problem through a reverberant layer, as depicted in Figure 1. We begin with considering a single sensor measurement, $y(t)$, that may contain flaw echoes, $s_{f}(t)$, and the reverberation clutter, $r(t)$, in the presence of additive measurement noise

$$
y(t)=s_{f}(t)+r(t)+n(t)
$$

where the noise $n(t)$ is zero-mean white Gaussian and is independent of the flaw echoes and reverberation clutter. Measurement noise is usually not considered as a serious impediment since its effect can be mitigated by averaging over multiple observations. The reverberation clutter, $r(t)$, is of quasiperiodic nature damped over time, where the periodicity and the degree of damping depend on the thickness and density of the layer. Under ideal measurement conditions (e.g., the layer is immersed in water in the far field of the transducer), the reverberation signal from the layer can be modeled as the superposition of the time-shifted and amplitude-scaled replicas of the transducer pulse echo wavelet, $s_{e}(t)$, as [1]

$$
r(t)=\rho s_{e}(t)+\varsigma_{12} \varsigma_{21} \sum_{m=1}^{\infty}(-\rho)^{2 m-1} s_{e}(t-2 m \Delta T),
$$

where $2 \Delta T$ denotes the time difference of arrival of successive echoes, $\rho$ denotes the reflection coefficient from the propagation path to the layer, and $\varsigma_{12}, \varsigma_{21}$ denote the transmission 


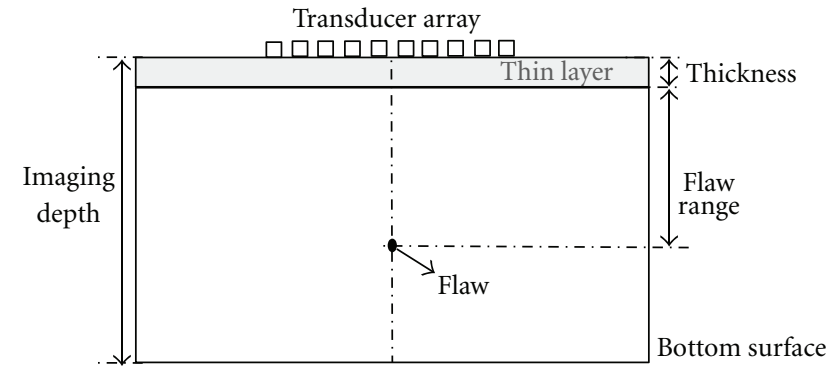

Figure 1: Experimental setup for flaw measurements through a reverberant layer.

coefficients from the propagation path to the layer and layer to the propagation path, respectively. This analytical reverberation model can be interpreted as superimposed echoes with unknown delays and amplitudes. Further, the reverberation signal can be estimated using a maximum likelihood estimation algorithm [13].

For contact measurements considered in this paper, the reverberation signal does not conform to the ideal model for a number of reasons. First, since the measurements are performed in the near field, the strong front-surface reflection (big bang) at the transducer-layer interface makes the reverberating pattern much more complicated. Second, the reverberation signal varies from one measurement to another depending on the coupling between the transducer and the layer as well as the bounding between the layer and the test material. On the other hand, the flaw echoes are short-duration signals with much lower energy compared to the reverberation clutter. Further, depending on the location of the flaw in the material, the flaw echo returns have longer time of arrival than those of the clutter. As such, the flaw echoes have a very low correlation with the reverberation clutter. Our objective is to remove the reverberation clutter without a considerable attenuation of flaw echoes. To this aim, we exploit the high energy and low correlation properties of the reverberation clutter with respect to the flaw echoes.

To model the reverberation clutter, we collect $L$ independent measurements of the clutter, $\mathbf{d}_{0}, \ldots, \mathbf{d}_{L-1}$, from $L$ different sites of a healthy replica of the material with layer, where $\mathbf{d}_{l}=\left[d_{l}(1), \ldots, d_{l}(N)\right]^{T}$ is the measured signal vector consisting of $N$ time samples and $(\cdot)^{T}$ denotes matrix transpose. These measurements are stored into an $N \times L$ matrix as

$$
\mathbf{Y}=\left[\begin{array}{llll}
\mathbf{d}_{0} & \mathbf{d}_{1} & \cdots & \mathbf{d}_{L-1}
\end{array}\right] .
$$

In the absence of flaw echoes, these measurements only contain reverberation clutter and noise. In order to account for local delays of reverberation, we expand these measurements based on a shift-based perturbation model [14]. Basically, all the measurement vectors are shifted by an integer number of samples, both upwards and downwards, up to a maximum potential delay of $d_{\max }$. Since ultrasound signals are typically sampled at a much higher rate than the Nyquist rate, shifting by integer samples will suffice to represent all possible local delays. With the time shifts, the expanded measurement set becomes

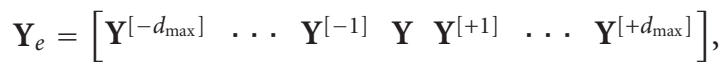

where $\mathbf{Y}^{[d]}$ is the original measurement matrix shifted by $d$ samples. The dimension of the expanded data set is $N \times M$ with $M=\left(2 d_{\max }+1\right) L$. The estimated covariance matrix of the expanded set $\mathbf{Y}_{e}$ is

$$
\mathbf{C}_{\mathbf{Y}_{e}}=\frac{1}{M} \mathbf{Y}_{e} \mathbf{Y}_{e}^{T}=\mathbf{C}_{r}+\sigma_{v}^{2} \mathbf{I},
$$

where $\mathbf{C}_{r}$ represents the clutter covariance matrix, $\sigma_{v}^{2} \mathbf{I}$ represents the covariance matrix of additive white Gaussian noise (AWGN), and $\mathbf{I}$ denotes the $N \times N$ identity matrix. Matrix $\mathbf{C}_{\mathbf{Y}_{e}}$ is decomposed into different spectral components using the eigenvalue decomposition

$$
\mathbf{C}_{\mathbf{Y}_{e}}=\sum_{m=1}^{M} \lambda_{m} \mathbf{u}_{m} \mathbf{u}_{m}^{T}
$$

where $\lambda_{m}$ denotes the $m$ th eigenvalue in an ordered set $\left(\lambda_{1} \geq \lambda_{2} \geq \cdots \geq \lambda_{M}\right)$ and $\mathbf{u}_{m}$ denotes the corresponding eigenvector. The first $\eta$ eigenvectors belong to the clutter subspace, whereas the remaining eigenvectors belong to the noise subspace. Since the dimension of the clutter subspace is not known a priori, model-order selection methods must be applied. The techniques found in the literature based on eigenvalue trend analysis such as the differences $\left(\lambda_{m}-\lambda_{m+1}\right)$ or ratios $\left(\lambda_{m} / \lambda_{m+1}\right)$ of eigenvalues do not always provide satisfactory results. We employ information theoretic criteria such as the Akaike information criterion (AIC) and the minimum description length (MDL) [15]. The AIC is given as

$$
\begin{aligned}
\operatorname{aic}(\eta)= & N \ln \left(\frac{\left[(1 /(M-\eta)) \sum_{m=\eta+1}^{M} \lambda_{m}\right]^{M-\eta}}{\prod_{m=\eta+1}^{M} \lambda_{m}}\right) \\
& +\eta(2 M-\eta),
\end{aligned}
$$

where $M$ denotes the number of measurements in the expanded data set. Similarly, the MDL is given as

$$
\begin{aligned}
\operatorname{mdl}(\eta)= & N \ln \left(\frac{\left[(1 /(M-\eta)) \sum_{m=\eta+1}^{M} \lambda_{m}\right]^{M-\eta}}{\prod_{m=\eta+1}^{M} \lambda_{m}}\right) \\
& +\frac{1}{2} \eta(2 M-\eta) \ln N .
\end{aligned}
$$

The clutter subspace is determined as the model order $\eta$ that minimizes the AIC or MDL. Once the model order is determined, the clutter subspace is formed from the first $\eta$ dominant eigenvectors, that is,

$$
\mathbf{U}_{r}=\left[\begin{array}{llll}
\mathbf{u}_{1} & \mathbf{u}_{2} & \cdots & \mathbf{u}_{\eta}
\end{array}\right] .
$$

The clutter removal is performed by projecting the received signal (A-scan) onto the orthogonal subspace of the clutter to obtain the flaw enhanced signal, that is,

$$
\widehat{\mathbf{s}}_{f}=\left(\mathbf{I}-\mathbf{U}_{r} \mathbf{U}_{r}^{T}\right) \mathbf{y} .
$$


2.2. Clutter Removal via Background Subtraction. For comparison, clutter removal via background subtraction is considered. Since a set of reference measurements are available for reverberation clutter, one can employ background subtraction techniques for clutter removal. For this task, we choose the best match signal from the set of expanded measurements matrix, $\mathbf{Y}_{e}$, to the current measurement $\mathbf{y}$ and subtract this signal from $\mathbf{y}$. The results represent the best possible performance that can be achieved with the background subtraction technique. In this case, the index of the best match reference signal is the one that maximizes the normalized correlation coefficient with $y$, that is,

$$
i_{m}=\arg _{i} \max \left|\frac{\mathbf{y}^{T} \mathbf{Y}_{e}[i]}{\sqrt{\left(\mathbf{y}^{T} \mathbf{y}\right)\left(\mathbf{Y}_{e}[i]\right)^{T}\left(\mathbf{Y}_{e}[i]\right)}}\right|
$$

where $\mathbf{Y}_{e}[i]$ denotes the $i$ th column of the expanded data set matrix $\mathbf{Y}_{e}$. The clutter removal using background subtraction is then performed as

$$
\widehat{\mathbf{s}}_{f}=\mathbf{y}-\left(\frac{\mathbf{y}^{T} \mathbf{Y}_{e}\left[i_{m}\right]}{\left(\mathbf{Y}_{e}\left[i_{m}\right]\right)^{T}\left(\mathbf{Y}_{e}\left[i_{m}\right]\right)}\right) \mathbf{Y}_{e}\left[i_{m}\right]
$$

where the scalar in the bracket term is the least square estimate of the best match reference signal amplitude.

\subsection{Clutter Removal via Predictive Deconvolution. For com-} parison, the predictive deconvolution technique $[3,4]$ is also considered for reverberation clutter removal. This technique has been extensively used in seismic signal processing for the suppression of reverberations due to the top layer of the earth [7]. The method is based on the linear prediction filtering and exploits the repetitive patterns in reverberations. The reverberating pattern is predicted based on the past samples of data. The method requires knowledge of the predictionlag (i.e., the periodicity of reverberation) as well as the pulse duration to set the predictive filter order and error filter length. In this paper, we implemented the technique based on the procedures outlined in [4].

\section{Clutter Removal Based on Sensor Array Measurements}

3.1. Clutter Removal. When the clutter-only scene is not available, the clutter subspace used for clutter removal must be constructed from data that are measured in the presence of flaw signals. Therefore, care must be exercised not to include the flaw signal in clutter subspace. Toward this end, we consider a $K$-element array, either consisting of physically present transducers or being formed through aperture synthesis, which measures the material of interest through a reverberant layer as depicted in Figure 1. The signal received at each sensor can be written as

$$
y_{k}(t)=\alpha_{k} s_{f}\left(t-\tau_{k}\right)+r(t)+n(t), \quad k=0,1, \ldots, K-1,
$$

where $y_{k}(t)$ denotes the signal received at the $k$ th sensor position, $r(t)$ denotes the reverberation clutter due to the layer, $\alpha_{k} s_{f}\left(t-\tau_{k}\right)$ denotes the echo signal received at the $k$ th sensor due to the flaw, and $n(t)$ denotes the AWGN. We note that the reverberation signal $r(t)$ is consistent across all sensor measurements except for local delays and small perturbations. On the other hand, flaw echo measurement varies from one sensor to another because each transducer position yields a distinct distance to the flaw. The variation in the flaw echo is modeled in terms of both the delays, $\left(\tau_{k}\right)$ due to the spatial arrangement of the array sensors, and the weighting factors $\left(\alpha_{k}\right)$, due to the beamwidth of the transducers and flaw reflection fluctuations. As a result, the reverberation measurements are spatially coherent, whereas flaw measurements are incoherent. In order to exploit the coherency as well as the relative strength of the reverberation, we utilize a subspace construction and projection approach.

For clutter subspace construction, we concatenate the sensor array measurements into a data matrix of size $N \times K$ as

$$
\mathbf{Y}_{a}=\left[\begin{array}{llll}
\mathbf{y}_{o} & \mathbf{y}_{1} & \cdots & \mathbf{y}_{K-1}
\end{array}\right]
$$

form the covariance matrix as

$$
\mathbf{C}_{\mathbf{Y}_{a}}=\frac{1}{N} \mathbf{Y}_{a} \mathbf{Y}_{a}^{T}
$$

and then perform eigendecomposition of the above estimated covariance matrix. This time, the dominant eigenvalues will correspond to the clutter subspace, followed by the eigenvalues corresponding to the flaw subspace and those corresponding to the noise subspace. Because the flaw signals are much weaker than the clutter, we can determine the clutter subspace using the AIC or MDL in the same manner as explained before. Finally, the clutter is removed from each sensor data by projecting onto the orthogonal subspace of the clutter to obtain flaw-enhanced signals on each sensor, that is,

$$
\hat{\mathbf{s}}_{f}^{[k]}=\left(\mathbf{I}-\mathbf{U}_{r} \mathbf{U}_{r}^{T}\right) \mathbf{y}_{k}
$$

Based on the clutter-free array data $\widehat{\mathbf{s}}_{f}^{[k]}$, an ultrasound image can be constructed for flaw imaging. For this task, we present a beamforming algorithm in the next section.

3.2. Ultrasound Imaging of Flaw via Beamforming. The ultrasound measurements from an array composed of $K$ transducers can be used to image the test material [16]. We consider a linear array and assume that the respective positions of transducer elements are known in a threedimensional Cartesian space, that is, the $k$ th transducer is located at $T_{k}\left(x_{T k}, y_{T k}, z_{T k}\right)$. We consider a region of interest (ROI), which is a two-dimensional cross-section under the linear array as depicted in Figure 1. A receiver mode backprojection beamforming algorithm is used to construct internal images of materials [17]. The signal that is reflected from a hypothetical flaw located at the position $P\left(x_{p}, y_{p}, z_{p}\right)$ is then received with different delays at each 
transducer. The signal corresponding to the direct reflection path recorded at the $k$ th receive transducer is given by

$$
r_{k}(t)=a_{k}(P) s_{e}\left(t-\tau_{k}(P)\right),
$$

where $a_{k}(P)$ is the reflectivity of the flaw seen by the transducer that also accounts for the propagation loss and $\tau_{k}(P)$ denotes the signal propagation delay from the location $P$ to the $k$ th transducer $T_{k}$. Assuming a homogeneous material with ultrasound propagation speed of $v$, the time delay corresponding to any pixel $Q$ in the image, located at $Q\left(x_{q}, y_{q}, z_{q}\right)$, can be calculated as

$$
\tau_{k}(Q)=\frac{2}{v}\left\|\left(x_{T m}, y_{T m}, z_{T m}\right)-\left(x_{q}, y_{q}, z_{q}\right)\right\|,
$$

where $\|\cdot\|$ denotes the Euclidean norm. The image intensity $I(Q)$ of every pixel $Q$ in the imaging plane is obtained by adding the weighted time-delayed $K$ received signals and correlating the resulting signal with the emitted signal. Therefore, the intensity at pixel $Q$, using the coherent imaging technique, can be written as

$$
\begin{aligned}
I(Q) & =\left.\sum_{k=1}^{K} w_{k}(Q) r_{k}\left(t+\tau_{k}(Q)\right) * s_{e}(t)\right|_{t=0} \\
& =\left.\sum_{k=1}^{K} w_{k}(Q) a_{k}(P) s_{e}\left(t+\tau_{k}(Q)-\tau_{k}(P)\right) * s_{e}(t)\right|_{t=0},
\end{aligned}
$$

where $w_{k}(Q)$ is the weight corresponding to the $k$ th transducer. The cross-correlation performs matched filtering and improves the output signal-to-noise ratio (SNR).

The above synthetic aperture beamforming algorithm is applied for imaging of flaws through layers before reverberation removal using the original array measurements and after reverberation removal using the proposed subspace projection approach. The flaw imaging results will be presented in the next section.

\section{Simulations and Experimental Results}

4.1. Experiment Settings. An aluminum block (alloy number $6061)$ of dimensions 6 in $\times 6$ in $\times 3$ in $(152.4 \mathrm{~mm} \times 152.4 \mathrm{~mm} \times$ $76.2 \mathrm{~mm}$ ) is used as the test specimen. A thin metal layer with $2 \mathrm{~mm}$ uniform thickness is coupled to the material with a gel. The thin layer is highly reverberant and simulates ultrasonic flaw imaging through reverberant layers. A flat-bottom hole with a diameter of $3 \mathrm{~mm}$ and a depth of $21 \mathrm{~mm}$ was drilled into the test specimen to emulate a flaw. Figure 1 shows the schematic illustration of the test specimen, the thin layer, and the synthesized sensor transducer used for ultrasonic measurements.

Transducer excitation and signal measurements are performed using an Olympus Panametrics Pulser/Receiver $(\mathrm{P} / \mathrm{R})$ (model 5072PR) operated in the monostatic (T/R) mode [18]. The P/R settings are as follows: pulse repetition frequency (PRF) $1 \mathrm{KHz}$, energy level 3, damping level 4, amplifier gain $30 \mathrm{~dB}$, low-pass filter with a cutoff frequency of $1 \mathrm{MHz}$, and high-pass filter with a cutoff frequency of $10 \mathrm{MHz}$. All the ultrasonic measurements are made with an Olympus single-element transducer (model V-110 M) that has a center frequency of $5 \mathrm{MHz}$ [19]. The transducer is placed on the material surface with an ultrasound coupling gel. The acquired signals are digitized with a digital scope (Agilent Technologies DSO7014A Oscilloscope) at a sampling rate of $50 \mathrm{MHz}$. These signals are collected 32 times and averaged internally by the scope to obtain a signal with a high SNR.

4.2. Simulation Results: Flaw-Free Measurements of Reverberation Clutter Available. We performed a series of simulations using synthetic data based on separate measurements of flaw and reverberation clutter to examine the respective clutter and flaw subspaces and analyze the performance of the subspace-projection-based clutter suppression technique. We first acquired a flaw signal measurement without the thin layer on top of the material. The flaw echo is properly truncated from this measurement and stored in memory. Next, the thin layer is placed on top of the aluminum block using an ultrasound gel as coupling. The reverberation measurement is performed by placing the $5 \mathrm{MHz}$ ultrasonic transducer on the thin layer and covering a healthy (flaw-free) section of the aluminum. We repeated this measurement on different sections of the thin layer for 10 times to obtain a diverse set of reverberation measurements representing clutter.

We used the 10 reverberation measurements with shiftbased subspace expansion $\left(d_{\max }=1\right)$ to form the clutter subspace as explained in Section 2. A new reverberation measurement (at a location different from the previous measurements) is made to test the clutter removal method. To examine the performance of the clutter removal technique under different conditions, we add the experimentally collected flaw echo and its multipath signals as if they were measured from the top layer to the new reverberation measurement but varied their amplitude and time of arrivals.

Figure 2 illustrates flaw echoes in reverberation and the clutter suppression results after respectively exploiting the subspace projection, background subtraction, and the predictive deconvolution techniques as described in Section 2. Figure 2(a) shows the emulated flaw echo and its multipath signals, whereas Figure 2(b) shows the reverberation measurement with the flaw echoes added. As such, the signal in Figure 2(b) simulates a flaw echo and its multipaths in reverberation. The flaw-echo-enhanced signals processed by the subspace projection, background subtraction, and predictive deconvolution techniques are, respectively, shown in Figures 2(c), 2(d), and 2(e). As evident from these plots, the proposed subspace projection technique highlights the flaw echoes and enhances their visibility. The background subtraction method, on the other hand, retains clutter remnants that can be mistaken as flaw echoes. The predictive deconvolution technique is also applied to this data with prediction lag set equivalent to the measured periodicity of reverberations and prediction filter length set equal to one echo length. The clutter suppression result (see Figure 2(e)) 


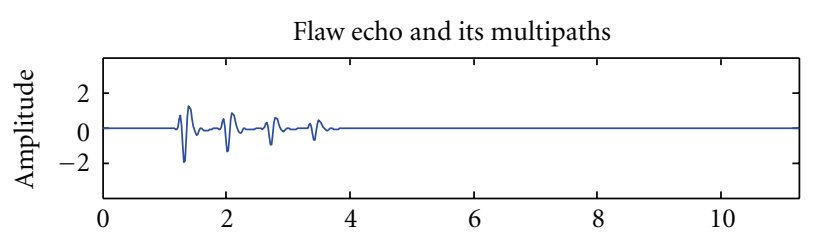

(a)

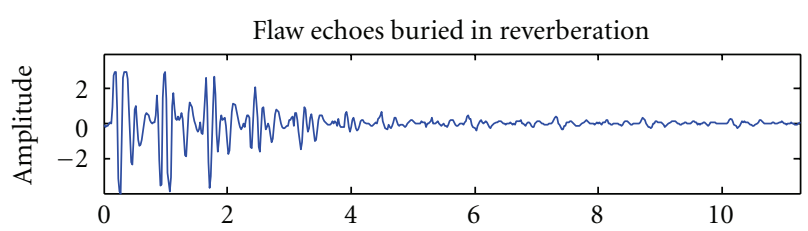

(b)

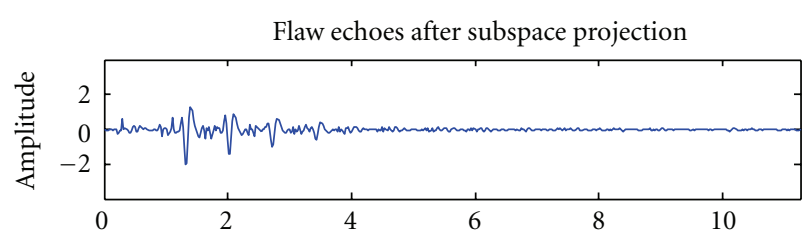

(c)

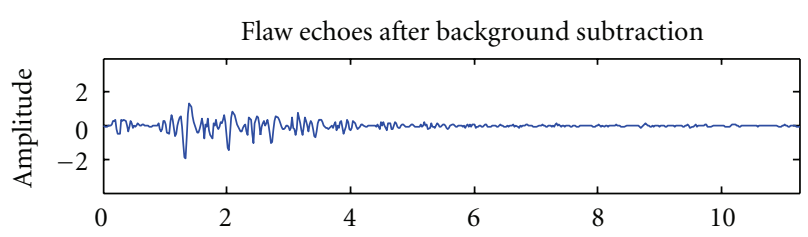

(d)

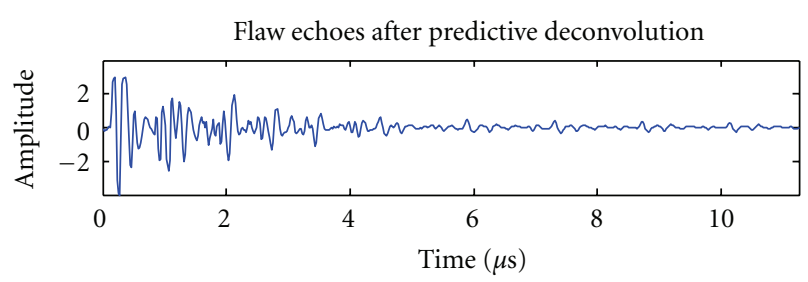

(e)

Figure 2: Comparison of reverberation removal techniques. (a) Simulated flaw echo and its multipaths measured through the layer. (b) Flaw echoes added to a real reverberation measurement. (c) Reverberation removal via subspace projection. (d) Reverberation removal via background subtraction. (e) Reverberation removal via predictive deconvolution.

is inferior to those obtained from background subtraction and subspace projection. This is due to the fact that, while the predictive deconvolution technique relies on the repeatability of reverberating patterns to predict and remove the future echoes, the actual reverberation echoes vary in their shape due to irregular variations and the existence of different propagation modes. For example, the noticeable difference in shape is observed between the first two echoes shown in Figure 2(b) during the time interval between $0 \mu$ s and $1.5 \mu$ s. Further, predictive deconvolution always retains the first part of data (e.g., the first echo in Figure 2(e)) before prediction lag. It is noteworthy that the proposed subspace projection
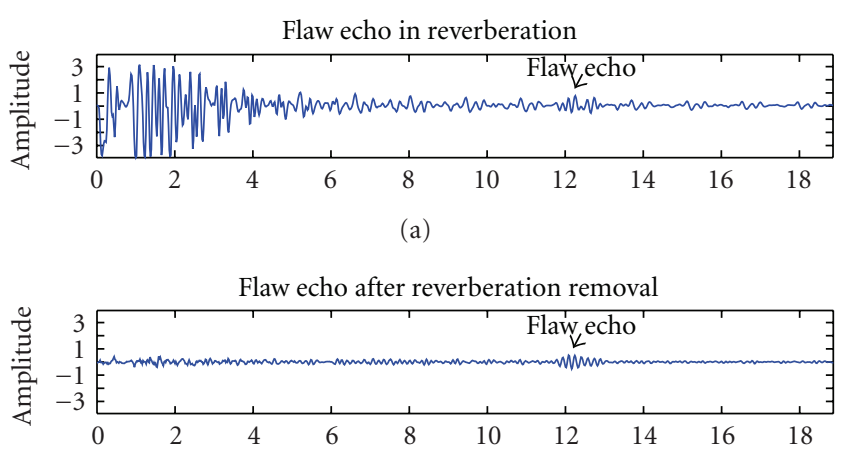

(b)

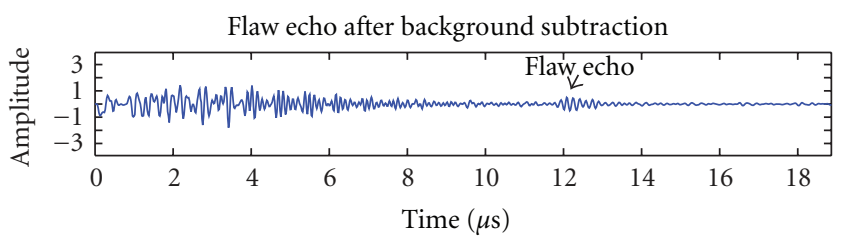

(c)

FIgURE 3: Reverberation removal using experimental data. (a) Ultrasonic measurement of the flaw through a reverberant layer. (b) Reverberation removal via subspace projection. (c) Reverberation removal via background subtraction.

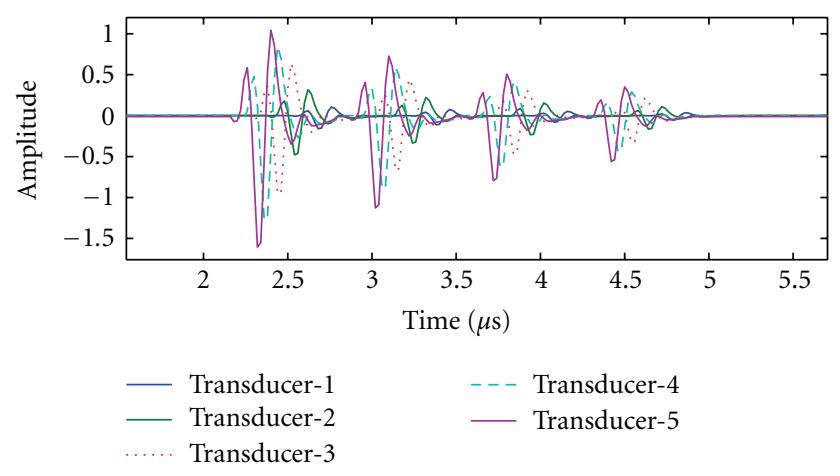

Figure 4: Simulation of flaw and its multipath measurements through the layer with a transducer array shown in Figure 1. The flaw is assumed to be in $1.35 \mathrm{~cm}$ depth from about the center of the array (see Figure 1).

technique works for arbitrary signal structures and does not rely on the repeatability of reverberation patterns.

We also made an ultrasonic measurement of a real flaw through the thin layer. To emulate a flaw, a through hole with a diameter of $3 \mathrm{~mm}$ is drilled into the aluminum block $3.5 \mathrm{~cm}$ down from the top. Then, the thin layer is placed on top of the block with gel coupling. The measurement including reverberations and the flaw echo is shown in Figure 3(a). The clutter-suppressed signals with subspace projection and background subtraction are shown in Figures 3(b) and $3(\mathrm{c})$, respectively. The subspace expansion technique with $d_{\max }=5$ samples is utilized. The model order is chosen based on the MDL metric. The flaw echo is located around 


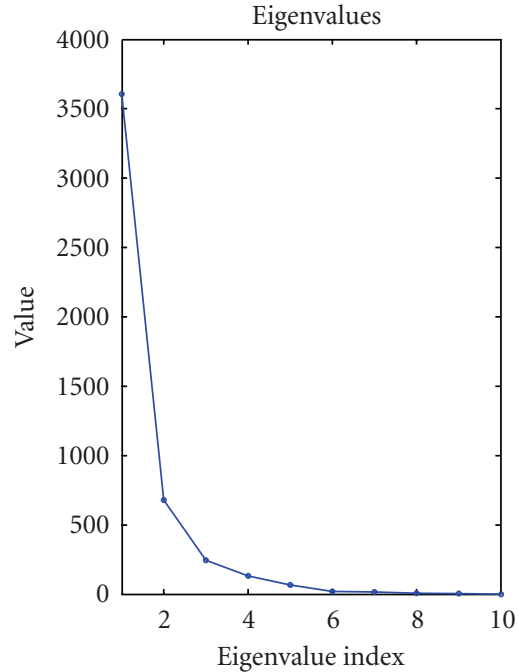

(a)

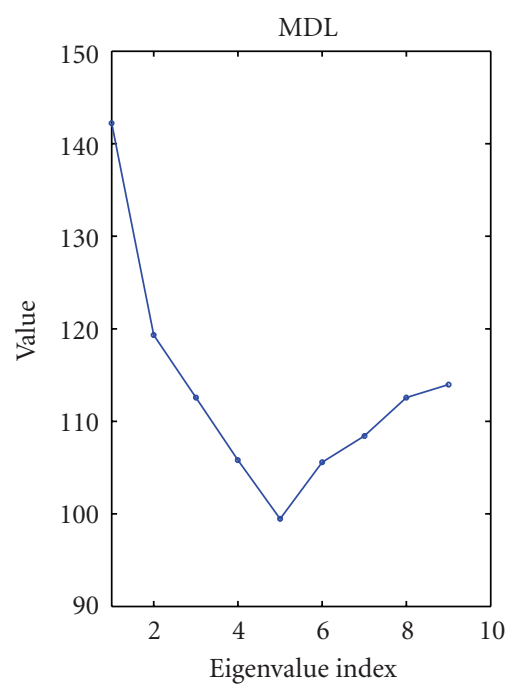

(b)

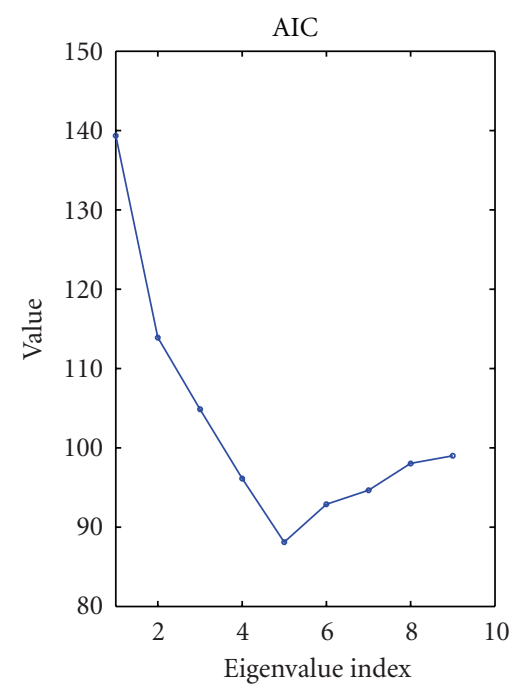

(c)

FIgURE 5: The eigenvalues (a) of the flaw in reverberation measurements with an array: the MDL (b) and AIC (c) metrics.

$12 \mu$ s. It is seen that the flaw echo is enhanced significantly after subspace-projection-based clutter removal, whereas background subtraction retains a significant portion of clutter.

\subsection{Simulations: Independent Measurement of Reverberation} Clutter Unavailable. In this section, we examine the reverberation removal technique when reference measurements of the flaw-free reverberation clutter are not available. This can be the case where there is no access to a healthy material. In order to allow clutter subspace estimation, we use multiple positions of the transducers, thus forming a synthetic array aperture that enables ultrasound imaging. By moving the transducer in collinear positions, it becomes equivalent to measuring the flaw with a linear array through the reverberant layer. As such, a subset or all measurements may contain flaw echoes, whereas all measurements contain the strong reverberation signal due to the layer. Clearly, clutter removal in this scenario is more challenging since reference measurements required to form a clutter subspace also contain flaw echoes.

We simulate an array measurement by moving the singleelement transducer in small steps on the surface of the thin layer placed on top of the material. To simulate flaw echoes impinged on reverberation measurements, the flaw echoes are generated based on the assumed flaw location, the geometry of the synthesized linear array, the respective delay between the flaw and sensors, and the approximate beamwidth of the measuring transducer. The beamwidth is incorporated as a weighting factor on echo amplitudes based on the flaw location with respect to transducer, as explained in [20]. Further, the flaw echo multipaths are simulated based on the assumed thickness and velocity of the layer and its reflection and transmission coefficients. A typical flaw echo and its multipath signals measured with the first 5 elements of the synthesized linear array (see Figure 1) are shown in Figure 4, where the flaw is located in the range of $1.35 \mathrm{~cm}$ from the array boresight. The flaw echo and its multipath signals received by the sensors close to the center are stronger and have a shorter time of arrival than those received by the sensors away from the center. To simulate the flaw measurement through a reverberant layer with a transducer array, we added these simulated flaw echoes to the 10 independent reverberation measurements obtained from the healthy sections of the aluminum block.

Because the flaw signal is contained in the measured waveforms that are used to construct the clutter subspace for orthogonal projection, it is important to accurately estimate the clutter subspace bases that are free of the flaw. As the reverberation clutter is orders of magnitude stronger than the flaw echoes, the clutter subspace can be limited to the eigenvectors associated with the dominant eigenvalues. In order to determine the dominant clutter subspace, we use information theoretic criteria, AIC and MDL, that were presented in Section 2. The flaw echo has a low correlation with the clutter and its energy is much smaller than the clutter energy. As such, its subspace is separate from the clutter subspace and is associated with smaller values of eigenvalues. The estimation of the flaw subspace dimension, however, is not necessary since clutter removal is sufficient to reveal the flaw echoes.

As seen from Figure 5, the dimension of the clutter subspace is chosen as the value that minimizes the AIC (7) or MDL (8) metric. In this case, both criteria provide the same clutter dimension estimate of 5 . The subspace-based clutter removal algorithm is tested on the synthetic data containing 10 reverberation measurements in the presence of flaw echoes. Figure 6(a) shows the simulated flaw echo and its multipath signals synthesized from data measured at the 4th transducer in the synthetic array (see Figure 1). Figure 6(b) shows the flaw echo and its multipath signals added to the reverberation. As such, Figure 6(b) simulates 


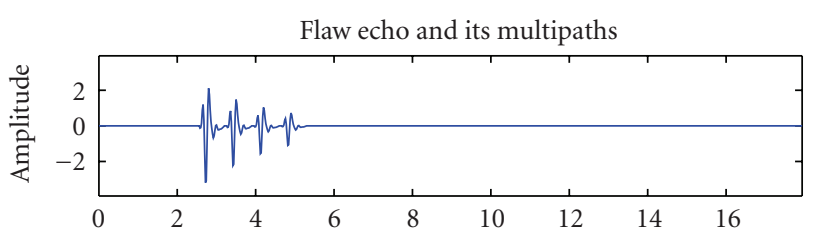

(a)

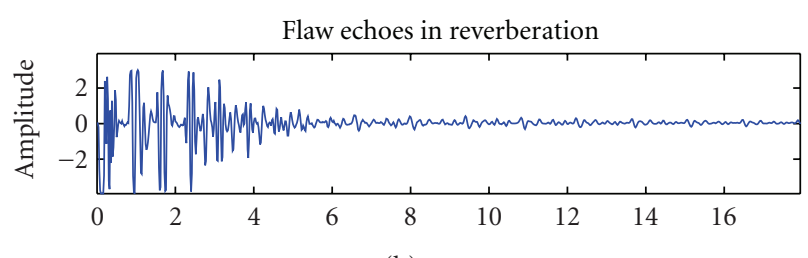

(b)

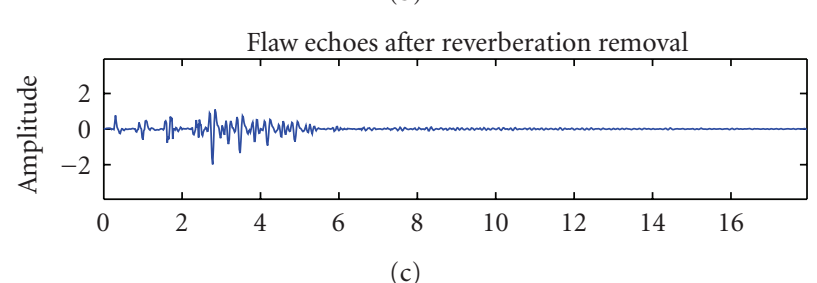

(c)

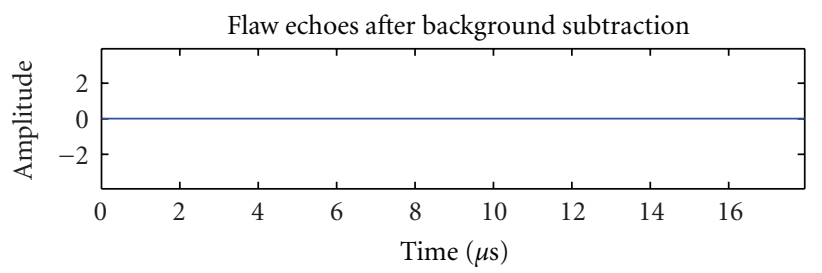

(d)

FIGURE 6: Reverberation removal based on array measurements containing the flaw echoes. (a) Simulated flaw echo and its multipaths received by the 4 th transducer shown in Figure 1. (b) Flaw echo and multipaths added to the reverberation measurement from the 4th transducer. (c) Reverberation removal via subspace projection. (d) Reverberation removal via background subtraction.

the flaw echo measurement with the 4th transducer through the thin layer. We apply the subspace projection technique with clutter subspace dimension set to 5 based on the MDL metric. The reverberation-suppressed signal is shown in Figure 6(c). Although the flaw echo and its multipath signals are clearly revealed, their amplitudes are smaller than the original (Figure 6(a)) owing to the fact that part of their energy lies in the clutter region. As expected, the subspace projection method based on the measurements of reverberations containing flaw echoes is less effective when the clutter and flaw waveforms overlap.

The background subtraction technique does not work for this case since flaw-free reference measurements of reverberation are not available. Direct application of background subtraction yields all-zero signal (Figure 6(d)) since the best match signal to the test signal (Figure 6(b)) is available in the reference set.

In another simulation, the flaw echo and its multipath signals are completely buried in reverberation (see Figure 7). This time, the flaw echo and its multipaths, as shown in

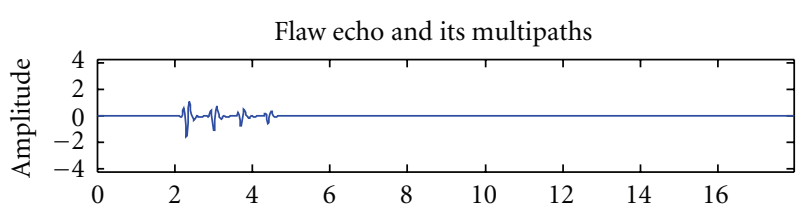

(a)

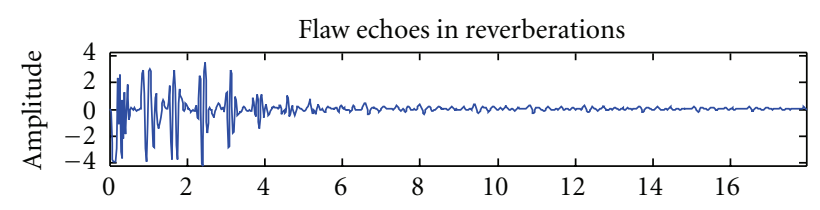

(b)

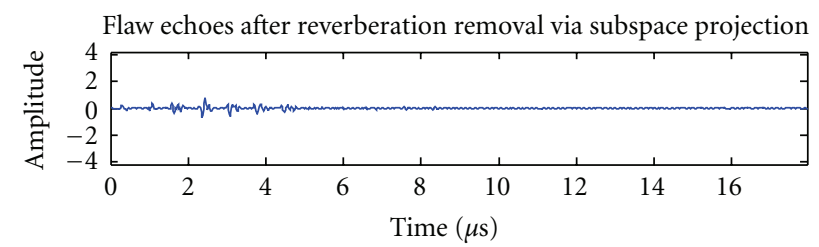

(c)

FIGURE 7: Reverberation removal in a high clutter area. (a) Simulated flaw echo and its multipaths received by the 4th transducer shown in Figure 1. (b) The flaw echo and its multipaths added to the reverberation measurement. The flaw echoes are completely buried in reverberation. (c) Reverberation removal via subspace projection.

Figure 7(a), are much weaker (the first flaw echo amplitude is set to 1) and buried in the dominant part of the reverberation signal as shown in Figure 7(b). As before, this signal is projected to the orthogonal subspace of the clutter with dimension 5 to obtain the flaw-enhanced echoes, as shown in Figure 7(c). The flaw echo and its multipath signals are visible albeit with smaller amplitudes than their original version. The remnants of the clutter are also visible but weaker than the first three flaw echoes.

Finally, we demonstrate the flaw imaging capability of the subspace projection technique in the presence of heavy clutter. For this purpose, we utilize the backprojection beamforming algorithm for synthetic aperture arrays as described in Section 3.1. The imaging results are depicted in Figure 8 . Figure 8 (a) shows the beamformed image of a flaw in the test material without the thin layer (see Figure 1) based on the simulated measurements of the flaw with the 10element synthetic array. Figure 8(b) shows the beamformed image when the flaw is measured through the layer. To simulate the array data associated with this image, the flaw and its multipath echoes, as measured through the layer, are added to the 10 independent reverberation measurements collected with the synthetic array. Finally, Figure 8(c) shows the beamformed image after the suppression of the reverberation clutter in the array data using the proposed subspace projection algorithm. The dimension of the clutter subspace is estimated as 5 using the MDL criterion. The positions of the 10-element synthetic arrays with respect to the flaw are shown in the top of the image. 


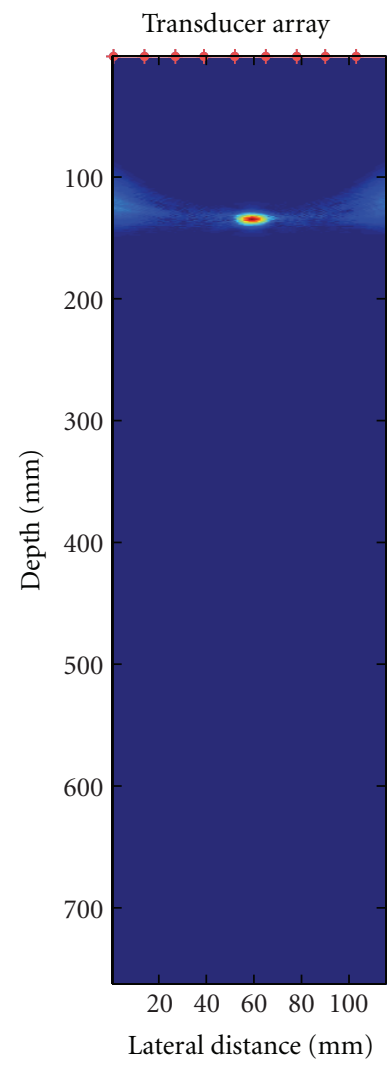

(a)

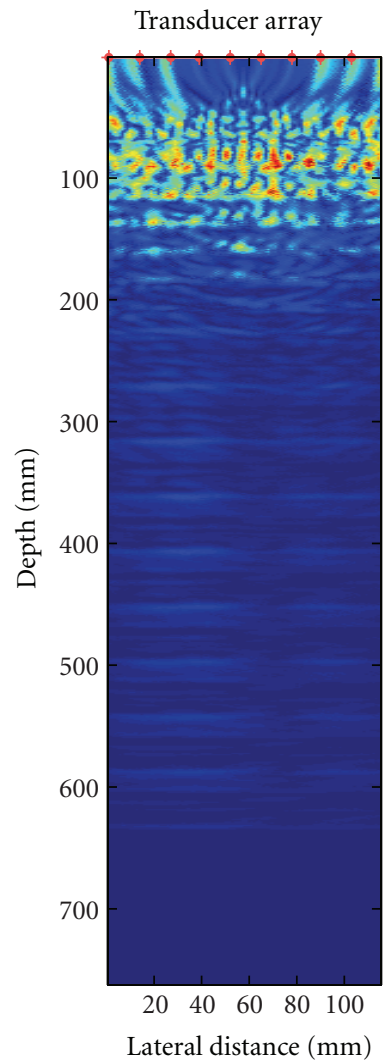

(b)

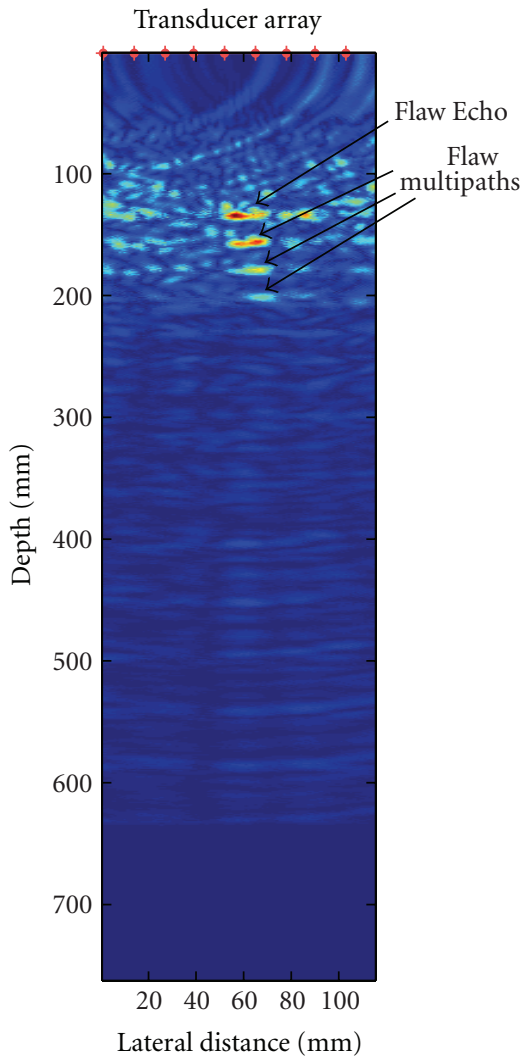

(c)

FIGURE 8: The synthetic aperture array imaging of a flaw through a thin reverberant layer. (a) Simulated flaw echo measurement with a 10-element synthetic linear array. The transducer positions are shown on top of the image. (b) Flaw (a) and its multipath measurements via the thin layer added to 10 reverberation measurements collected with the synthetic array. (c) Imaging after reverberation removed array data. The flaw echo and its multipaths are clearly revealed.

It is evident from Figure 8 that the proposed clutter removal algorithm effectively suppresses the clutter and significantly enhances the visibility of the flaw echo and its first few multipaths, which otherwise are buried in reverberation. Further, the technique is very practical since it operates on the existing array data and adds a negligible computational complexity to the synthetic aperture beamforming imaging algorithm and hence can be incorporated into real-time ultrasound imaging systems.

\section{Conclusions}

In this paper we proposed a subspace learning and projection technique for suppression of reverberation signals that arise in detection and imaging of flaws through layers. We addressed reverberation clutter removal in ultrasound nondestructive evaluation (NDE) in the presence of very strong reverberation. Two different cases are considered for the application of the proposed technique. The first case uses a set of flaw-free reference measurements of reverberation, whereas the second case is based on array measurements that contain flaws. For the first case, we have shown that, by utilizing a set of reference reverberation signals obtained from the healthy replica of the materials, the clutter can be significantly removed with a negligible effect on the flaw echoes. In the second case that applies to ultrasound NDE imaging, we demonstrated that the clutter can be mitigated by utilizing the array measurements without employing any reference data. The effectiveness of the proposed clutter removal techniques for both cases is verified by analysis and experimental data. The proposed clutter removal technique is computationally efficient and practical for array imaging. It does not require any parameter tuning or the knowledge of the screening layer propagation characteristics. As such, it can be easily incorporated into the existing array imaging systems with minimal complexity.

\section{Acknowledgments}

This research is supported in part by the National Science Foundation (NSF) under Grant no. IIP-0917690 and by The Ministry of Railways, China, under Grant no. 2011J011-E.

\section{References}

[1] J. Saniie and D. T. Nagle, "Pattern recognition in the ultrasonic imaging of reverberant multilayered structures," IEEE Transac- 
tions on Ultrasonics, Ferroelectrics, and Frequency Control, vol. 36, no. 1, pp. 80-92, 1989.

[2] S. W. Smith, G. E. Trahey, and O. T. Von Ramm, "Phased array ultrasound imaging through planar tissue layers," Ultrasound in Medicine and Biology, vol. 12, no. 3, pp. 229-243, 1986.

[3] D. Kishoni, "Removal of dominant reverberations from ultrasonic time-records of layered material by digital predictive deconvolution," in Proceedings of the IEEE Ultrasonics Symposium, pp. 1075-1078, 1986.

[4] Y. F. Chang, Y. Ma, C. M. Lin, and J. H. Lee, "Reverberation reduction in ultrasonic images via predictive deconvolution," NDT\&E International, vol. 41, no. 4, pp. 235-241, 2008.

[5] M. A. Duarte, J. C. Machado, and W. C. A. Pereira, "A method to identify acoustic reverberation in multilayered homogeneous media," Ultrasonics, vol. 41, no. 9, pp. 683-698, 2004.

[6] K. K. Win, J. Wang, C. Zhang, and R. Yang, "Identification and removal of reverberation in ultrasound imaging," in Proceedings of the 5th IEEE Conference on Industrial Electronics and Applications (ICIEA '10), pp. 1675-1680, June 2010.

[7] O. Yilmaz, Seismic Data Analysis: Processing, Inversion, and Interpretation of Seismic Data, Society of Exploration Geophysics, Tulsa, Okla, USA, 2000.

[8] A. M. Mayordomo and A. Yarovoy, "Optimal background subtraction in GPR for humanitarian demining," in Proceedings of the 5th European Radar Conference (EuRAD '08), pp. 48-51, October 2008.

[9] Y-S. Yoon and M. G. Amin, "Spatial filtering for wallclutter mitigation in through-the-wall radar imaging," IEEE Transactions on Geoscience and Remote Sensing, vol. 47, no. 9, pp. 3192-3208, 2009.

[10] F. H. C. Tivive, A. Bouzerdoum, and M. G. Amin, "An SVDbased approach for mitigating wall reflections in through-thewall radar imaging," in Proceedings of IEEE Radar Conference, Kansas City, Kan, USA, May 2011.

[11] V. Kovalenko, A. G. Yarovoy, and L. P. Ligthart, "A novel clutter suppression algorithm for landmine detection with GPR," IEEE Transactions on Geoscience and Remote Sensing, vol. 45, no. 11, pp. 3740-3750, 2007.

[12] B. W. Drinkwater and P. D. Wilcox, "Ultrasonic arrays for nondestructive evaluation: a review," NDT\&E International, vol. 39, no. 7, pp. 525-541, 2006.

[13] R. Demirli and J. Saniie, "Model-based estimation of ultrasonic echoes part II: nondestructive evaluation applications," IEEE Transactions on Ultrasonics, Ferroelectrics, and Frequency Control, vol. 48, no. 3, pp. 803-811, 2001.

[14] X. Shen, Y. D. Zhang, M. G. Amin, and R. Demirli, "Ultrasound multipath background clutter mitigation based on subspace analysis and projection," in Proceedings of the IEEE International Conference on Acoustics, Speech and Signal Processing (ICASSP '12), March 2012.

[15] M. Wax and T. Kailath, "Detection of signals by information theoretic criteria," IEEE Transactions on Acoustics, Speech, and Signal Processing, vol. 33, no. 2, pp. 387-392, 1985.

[16] F. Ahmad, M. G. Amin, and S. A. Kassam, "Synthetic aperture beamformer for imaging through a dielectric wall," IEEE Transactions on Aerospace and Electronic Systems, vol. 41, no. 1, pp. 271-283, 2005.

[17] M. G. Amin, Ed., Through-the-Wall Radar Imaging, CRC Press, 2010.

[18] Olympus Ultrasonic Pulser/Receiver, http://www.olympusims.com/en/5072pr/.

[19] Olympus NDT contact transducers, http://www.olympusims.com/en/ultrasonic-transducers/contact-transducers/.
[20] R. Demirli, X. Rivenq, Y. D. Zhang, C. Ioana, and M. G. Amin, "MIMO array imaging for ultrasonic nondestructive testing," in Nondestructive Characterization for Composite Materials, Aerospace Engineering, Civil Infrastructure, and Homeland Security, Proceedings of SPIE, San Diego, Calif, USA, March 2011. 

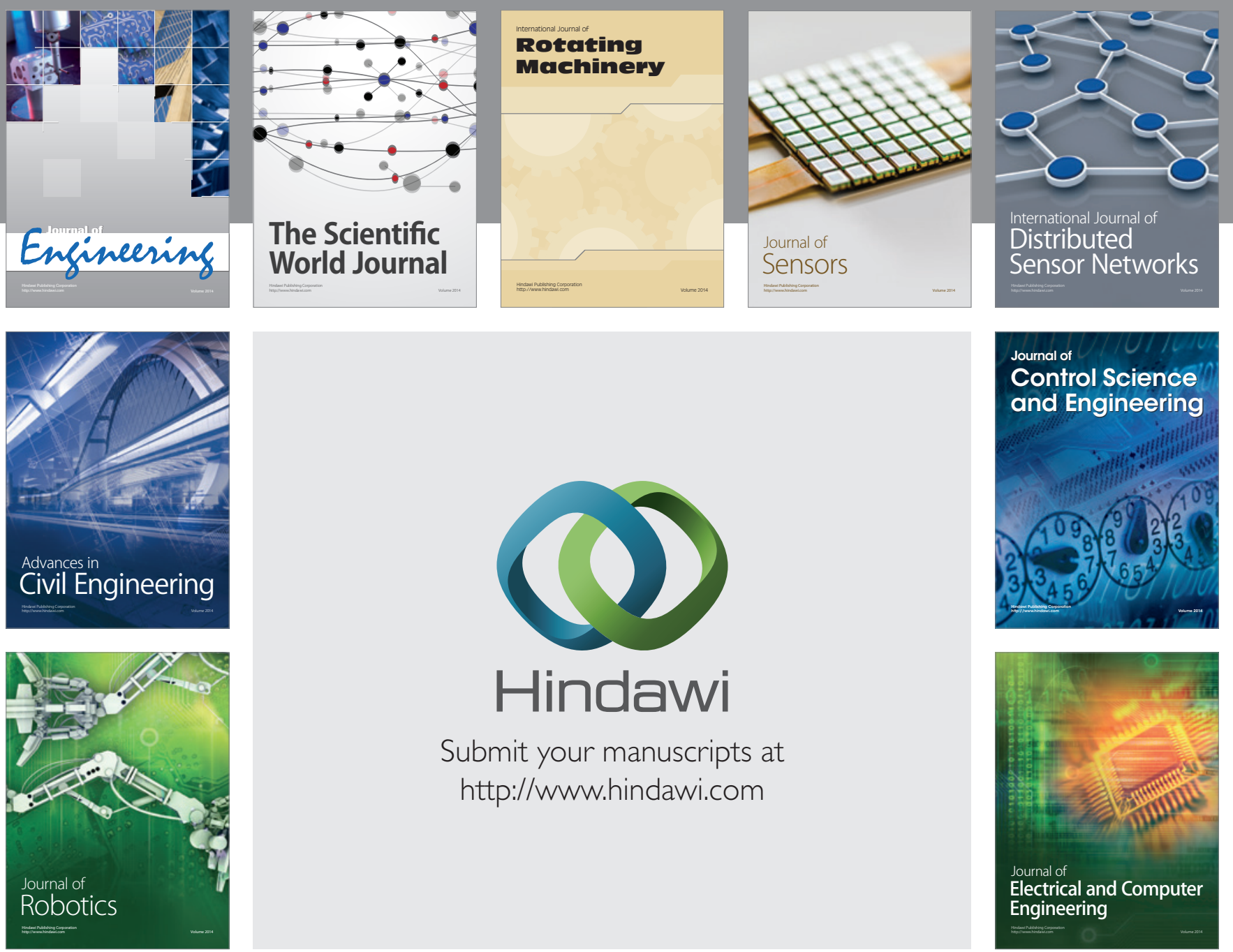

Submit your manuscripts at

http://www.hindawi.com
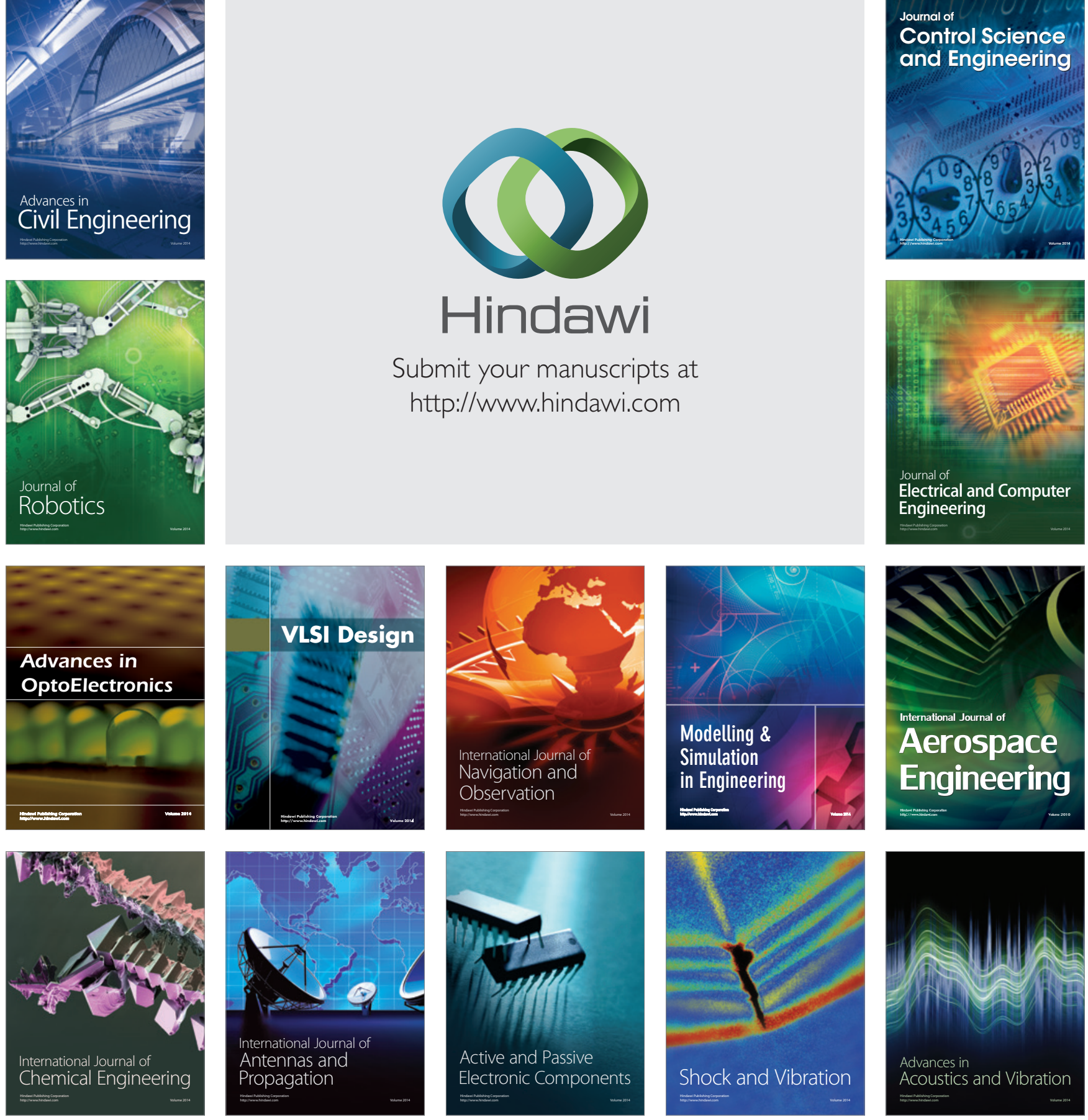